\title{
Estudio de los factores que afectan a la velocidad de neutralización de la pulpa durante la elaboración de aceitunas tipo negras
}

\author{
Por M. Brenes, P. García, C. Romero y A. Garrido. \\ U.E.I. de Biotecnología de Alimentos. \\ Instituto de la Grasa y sus Derivados. Aptdo. 1078, Sevilla (Spain).
}

RESUMEN

Estudio de los factores que afectan a la velocidad de neutralización de la pulpa durante la elaboración de aceitunas tipo negras.

El trabajo estudia la influencia de varios factores sobre la velocidad de neutralización de la pulpa de aceitunas tipo negras durante la etapa de ennegrecimiento. De los agentes neutralizantes estudiados (ácido clorhídrico y anhídrido carbónico), el $\mathrm{CO}_{2}$ ha mostrado una considerable mayor efectividad; el aumento de la dureza del agua empleada en los lavados disminuye la velocidad de neutralización. Finalmente, la temperatura tiene también una influencia beneficiosa, aunque nunca llega a superar el efecto del empleo de $\mathrm{CO}_{2}$. Estos resultados permiten diseñar este proceso de neutralización de forma más racional y eficaz, lo que, a su vez, puede dar lugar a reducir significativamente el volumen de vertidos que actualmente se producen en esta fase.

PALABRAS-CLAVE: Aceítuna tipo negra - Elaboración - Neutralización - Pulpa.

\section{SUMMARY}

Study of the factors affecting the rate of neutralization of olive flesh during the production of black olive.

The present work investigates the influence of various factors on the rate of neutralization of black olive flesh during the blackening stage. Of the neutralizing agents studied (hydrochloric acid and carbon dioxide), $\mathrm{CO}_{2}$ proved to be much more effective. Washing with harder water lessened the rate of neutralization. In addition a rise in temperature also exerts a beneficial influence, although heating never produces a better effect than the use of $\mathrm{CO}_{2}$. These results allow for a more rational and effective neutralizing process to be developed. This, in turn, could lead to a significant reduction in the amount of waste produced in this phase under present practices.

KEY-WORDS: Flesh - Neutralization - Processing - Ripe olive.

\section{INTRODUCCION}

La elaboración de aceitunas tipo negras se realiza según un procedimiento iniciado y desarrollado en USA a principios de siglo (Cruess, 1958). Los frutos se tratan con sucesivas soluciones de $\mathrm{NaOH}$, sumergiéndose en una solución acuosa en la que se inyecta aire en el intervalo entre los tratamientos alcalinos. A lo largo de este proceso se produce un paulatino ennegrecimiento de la piel y, en menor medida, de la pulpa debido a la transformación de los ortodifenoles (hidroxitirosol y ácido cafeico) presentes en las aceitunas (Brenes et al, 1992).

En el último tratamiento con hidróxido sódico, éste penetra hasta el hueso con lo cual la pulpa de los frutos alcanza un $\mathrm{pH}$ de, aproximadamente, unas 12 unidades. La eliminación del exceso de álcali se efectúa actualmente mediante lavados con agua a la que se adicionan ácidos minerales como sulfúrico (McCorkle et al., 1984) o clorhídrico (Fernández et al., 1985). Estos ácidos tienen el inconveniente de originar en las aceitunas sabores extraños cuando las cantidades utilizadas son elevadas (Miller, 1979). Para evitar estos inconvenientes se ha propuesto el uso de anhídrido carbónico como agente neutralizante, ya que el pH de la pulpa sólo debe quedar alrededor de 7,0 unidades, por lo que no es imprescindible la utilización de ácidos fuertes. Con ello, además, se suprime la presencia de cloruros y sulfatos en las aguas de lavado, lo que puede representar un obstáculo para el vertido de las mismas (Moffat, 1974). La neutralización con $\mathrm{CO}_{2}$ se lleva a cabo saturando con dicho gas la solución en la que están suspendidas las aceitunas (Scrimstrire, 1987).

Por otra parte, la velocidad de neutralización de la pulpa debe afectarse por una serie de variables aún no estudiadas en el caso de las aceitunas tipo negras. En las tipo verdes se ha comprobado que el empleo de aguas duras retrasa la penetración del hidróxido sódico durante el "cocido" (Rodríguez de la Borbolla et al.,1956), por lo que es de esperar que la concentración de calcio y magnesio pueda influir en todos estos procesos de intercambio osmótico fruto/salmuera. Asimismo, el incremento de la temperatura favorece dichos fenómenos, lo que debe representar una mayor velocidad de neutralización.

El presente trabajo tiene el objetivo de conocer mejor los factores que influyen en la fase de neutralización del exceso de álcali en el transcurso de la elaboración de aceitunas tipo negras y así poder diseñar el mismo de una manera más racional. Las variables estudiadas son: tipo de agente neutralizante $\left(\mathrm{HCl}\right.$ ó $\left.\mathrm{CO}_{2}\right)$, dureza del agua de lavado y temperatura de trabajo. 


\section{PARTE EXPERIMENTAL}

\subsection{Aceitunas}

Se emplean aceitunas Hojiblancas (Olea europaea arolensis) conservadas durante un periodo de 2 a 5 meses en salmuera.

\subsection{Equipos}

Las experiencias se realizan en 4 recipientes cilíndricos de $0,3 \mathrm{~m}$ de alto y $0,15 \mathrm{~m}$ de diámetro con la base inferior cónica por cuyo vértice se introduce aire a razón de 0,3 $\mathrm{m}^{3} / \mathrm{h}$ (García et al., 1991).

El pH del líquido durante la neutralización se controla mediante un equipo Crison, $\mathrm{pH} / \mathrm{mv}$ controller 252 (Crison, Barcelona, España), y la temperatura se mantiene en los valores fijados gracias a un calentador de agua automático Rena (Rena Corporation, Charlotte, USA).

\subsection{Realización de las experiencias}

Se colocan a temperatura ambiente $\left(17-20^{\circ} \mathrm{C}\right) 2 \mathrm{~kg}$ de aceitunas y 2 I de solución $0,5 \mathrm{M}$ de $\mathrm{NaOH}$, dejándose el tiempo necesario, unas 8 horas, para que el hidróxido penetre hasta el hueso. Seguidamente, se retira la lejía y se coloca, excepto en los casos en los que en el diseño así se haga constar, en $2 \mathrm{I}$. de agua de la red urbana de Sevilla, manteniéndose el pH del líquido a 8,0 ó 6,5 unidades mediante la adición controlada de $\mathrm{CO}_{2}$ ó solución $0,4 \mathrm{M}$ de $\mathrm{HCl}$.

Periódicamente se toman muestras de aceitunas para determinar el $\mathrm{pH}$ de la pulpa.

\subsection{Análisis}

El pH de la pulpa de los frutos se determina en la pasta resultante de triturar 10 aceitunas (unos $30 \mathrm{~g}$ ) con un triturador Ultraturrax, mediante un potenciómetro Beckman modelo 45.

Para la realización de la curva tampón se toman unos $300 \mathrm{~g}$ de frutos y una vez que la lejía ha penetrado hasta el hueso, se deshuesan y trituran. La pasta resultante se filtra a través de papel (Albet $n^{\circ}$ 305) obteniéndose un jugo con el que se realiza la valoración potenciométrica con un equipo Metrohm 670 (Metrohm, Herisau, Suiza), tal como describen Fernández y González (1956).

\subsection{Diseño de las experiencias}

El efecto de los dos agentes neutralizantes ensayados $\left(\mathrm{HCl}\right.$ y $\left.\mathrm{CO}_{2}\right)$ se ha estudiado a dos niveles de $\mathrm{pH}$ del líquido, 6,5 y 8,0 unidades, utilizándose un diseño factorial $2^{2}$. En todos los casos el agua empleada en los lavados ha sido agua potable de la red urbana con una concentración de $35 \mathrm{ppm}$ de $\mathrm{Ca}^{++}$. Las experiencias se han efectuado a temperatura ambiente $\left(20^{\circ} \mathrm{C}\right)$.

La influencia de la dureza se ha investigado usando como soluciones de lavado agua destilada con 0,50 y 200 ppm de $\mathrm{Ca}^{++}$Para el estudio de la interacción con el tipo de acidulante $\left(\mathrm{HCl}\right.$ ó $\left.\mathrm{CO}_{2}\right)$ se plantea una experiencia factorial $2^{2}$ con replicación, en la que los líquidos empleados son agua destilada y agua de la red con $80 \mathrm{ppm}$ de $\mathrm{Ca}^{++}$. El pH del agua durante el lavado se fija a 6,5 unidades y la temperatura ambiente fue de $17^{\circ} \mathrm{C}$.

Finalmente se ha estudiado la neutralización con $\mathrm{HCl}$ y $\mathrm{CO}_{2}$ a diferentes temperaturas $\left(10,20,30,40\right.$ y $\left.50^{\circ} \mathrm{C}\right)$, utilizando agua de la red con $35 \mathrm{ppm}$ de $\mathrm{Ca}^{++}$y fijándose el $\mathrm{pH}$ a 6,5 unidades.

\section{RESULTADOS Y DISCUSION}

\subsection{Curva de capacidad amortiguadora}

Las variaciones del $\mathrm{pH}$ cuando se añaden ácidos o bases dependen de la capacidad tampón de la solución correspondiente. Por ello, es básico conocerla previamente. En la Figura 1 se recoge la curva de índice tampón del jugo de aceitunas una vez realizado el tratamiento con $\mathrm{NaOH}$. De su estudio destaca el hecho de que por encima de un $\mathrm{pH}$ de 11 unidades se tienen los valores más altos, necesitándose, por tanto, una mayor cantidad de ácido para disminuir el $\mathrm{pH}$ en el mismo intervalo. Entre 11 y 9 los valores son menores y bastante similares, incluso con un pequeño máximo a 9,5 unidades. Por debajo de 9 disminuye rápidamente, lo que implica que pequeñas cantidades de ácido son suficientes para llevar el $\mathrm{pH}$ a valores en torno a las 7,0 unidades.

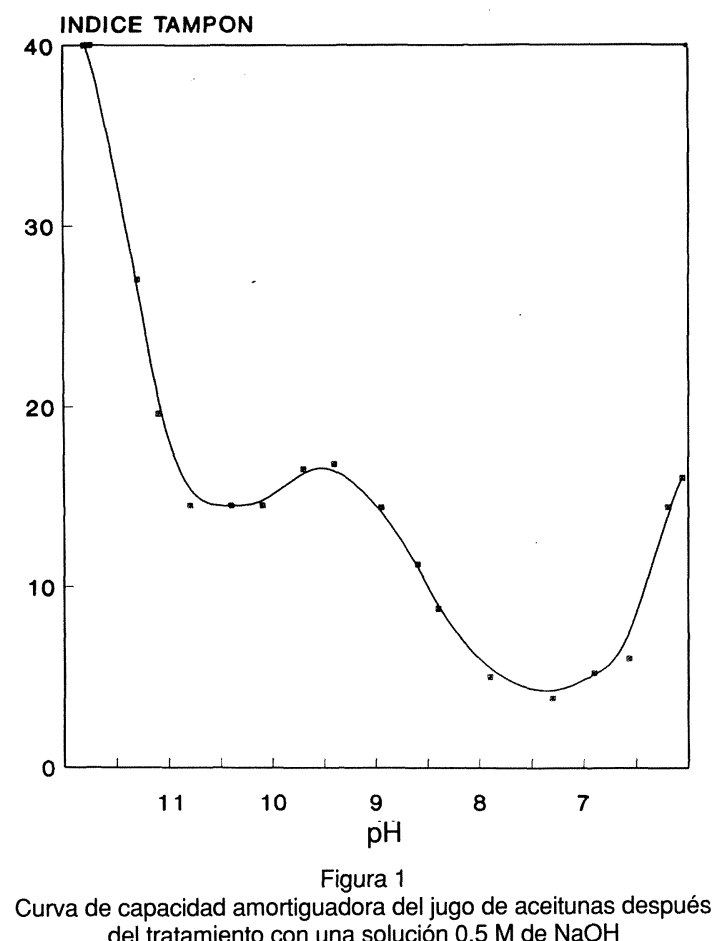
del tratamiento con una solución $0,5 \mathrm{M}$ de $\mathrm{NaOH}$ 
Según ello, la neutralización de la pulpa de aceitunas tipo negras después del último tratamiento con $\mathrm{NaOH}$ debe acelerarse una vez que se alcance un $\mathrm{pH}$ de 9 unidades debido a la existencia de una débil capacidad amortiguadora por debajo de dicho valor.

\subsection{Efecto del agente neutralizante $\left(\mathrm{CO}_{2}\right.$ ó $\left.\mathrm{HCl}\right)$}

La evolución del $\mathrm{pH}$ de la pulpa de aceitunas cuando se realiza la neutralización mediante adición de $\mathrm{CO}_{2}$ ó $\mathrm{HCl}$ con la ayuda del equipo automático de control se recoge en la Figura 2. Como se observa, para el mismo acidulante el descenso del pH de la pulpa es más acusado cuando el líquido se mantiene a un $\mathrm{pH}$ inferior (6,5 unidades) debido al mayor gradiente de $\mathrm{pH}$ que se crea entre la pulpa y el líquido.

Es de destacar que la disminución del $\mathrm{pH}$ cuando se emplea $\mathrm{CO}_{2}$ es mucho más rápida que con $\mathrm{HCl}$, alcanzándose en el primer caso en sólo 6 horas de tratamiento un $\mathrm{pH}$ de 8 unidades en la pulpa. Sin embargo, para el mismo tiempo, con $\mathrm{HCl}$ se mantienen aún valores superiores a 10,5 unidades. Esta diferencia se debe a la mayor velocidad de difusión del gas frente al ácido inorgánico en el interior de la pulpa de las aceitunas cuando se alcanza la saturación en el líquido.

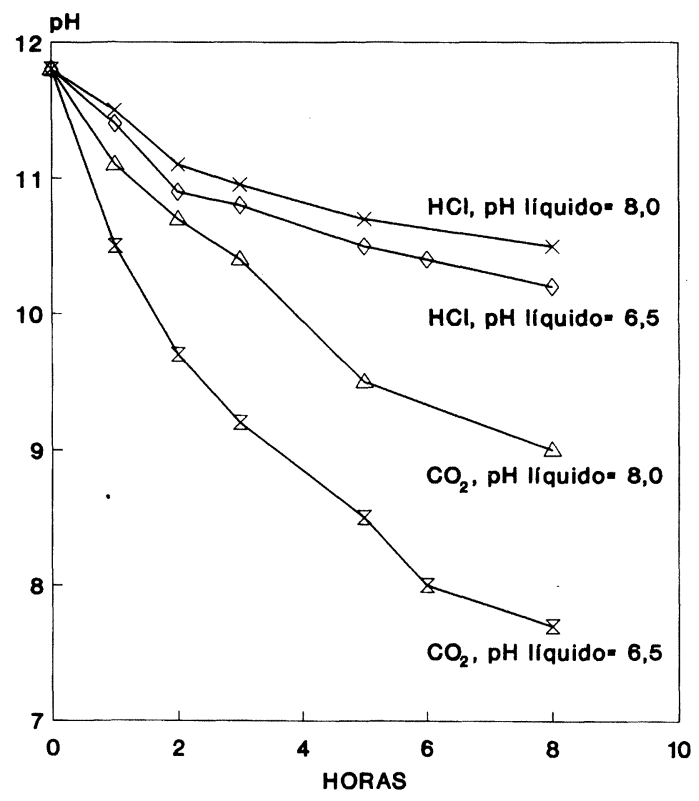

Figura 2

Evolución del pH de la pulpa de aceitunas según el agente neutralizante empleado $\left(\mathrm{HCl}\right.$ ó $\mathrm{CO}_{2}$ ) y el pH fijado del líquido (6,5 y 8,0 unidades). El lavado se realizó con agua de la red con $35 \mathrm{ppm}$ de $\mathrm{Ca}^{++} \mathrm{a}$ una temperatura de $20^{\circ} \mathrm{C}$

\subsection{Efecto de la dureza del agua de lavado}

Como se observa en la Figura 3, el emplear aguas con distinto contenido en $\mathrm{Ca}^{++}$implica que desde los primeros lavados se tengan diferencias significativas en los valores del $\mathrm{pH}$ de la pulpa.

Los $\mathrm{pH}$ más bajos, que indican una mayor velocidad de neutralización, se corresponden con el caso en que se emplea sólo agua destilada. En las condiciones de la experiencia, cuando el contenido en $\mathrm{Ca}^{++}$es de 50 ó 200 ppm es necesario para alcanzar un $\mathrm{pH}$ de 9,5 unidades, que permita el paso a la fase de fijación del color en la elaboración, realizar un lavado más de 12 ó 24 horas respectivamente que cuando se utiliza agua exenta de $\mathrm{Ca}^{++}$, tal como se desprende del estudio de la Figura 3.

\subsection{Interacción agente neutralizante y dureza del agua de lavado}

Los resultados de la experiencia realizada, recogidos en la Figura 4, reafirman las conclusiones de los dos anteriores apartados: el emplear aguas menos duras y anhídrido carbónico en vez de $\mathrm{HCl}$ aceleran el proceso de neutralización de la pulpa de aceitunas.

En esta experiencia cuando se emplea agua de la red se observa una menor velocidad de neutralización que la obtenida en la Figura 2, tanto para el $\mathrm{HCl}$ como para el $\mathrm{CO}_{2}$, debido al mayor contenido en sales en el caso de la Figura 4 (particularmente en $\mathrm{Ca}^{++}, 80 \mathrm{ppm}$ frente a $35 \mathrm{ppm}$ ) y a la menor temperatura de trabajo $\left(17^{\circ} \mathrm{C}\right.$ frente a $\left.20^{\circ} \mathrm{C}\right)$.

3.5. Efecto de la temperatura y su interacción con el tipo de agente neutralizante

Como se observa en la Figura 5, cuando se aumenta la

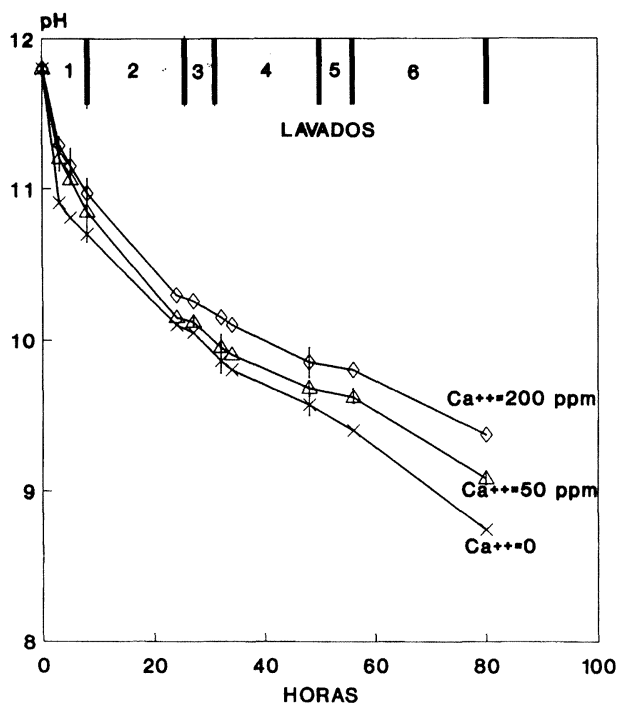

Figura 3

Evolución del pH de la pulpa de aceitunas durante los lavados con aguas de distinto contenido en $\mathrm{Ca}^{++}$. Temperatura $20^{\circ} \mathrm{C}$. Cuando no se observan las barras de error, las determinaciones están dentro del intervalo del símbolo. 
temperatura del líquido se produce un incremento en la velocidad de neutralización, tanto si se emplea ácido clorhídrico como anhídrico carbónico.

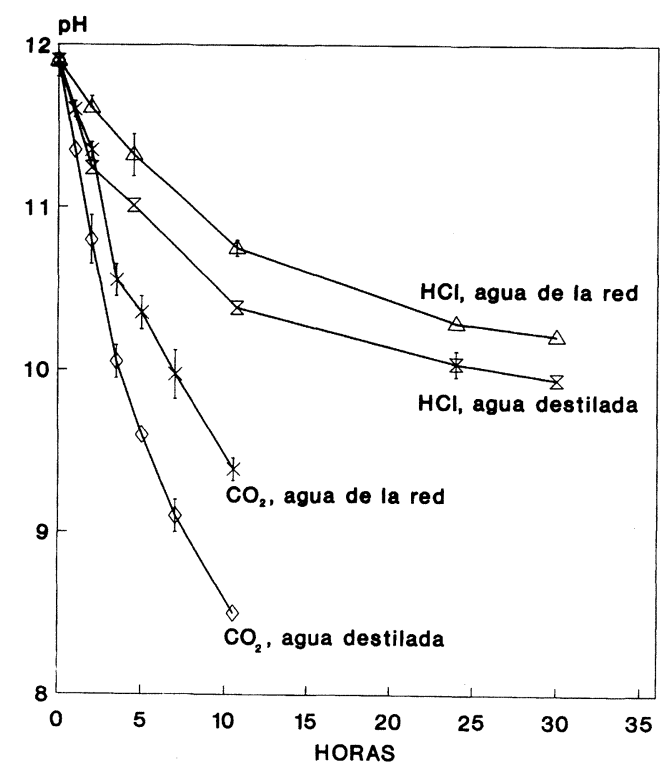

Figura 4

Evolución del pH de la pulpa de aceitunas según el agente neutralizante empleado $\left(\mathrm{HCl}_{\text {ó }} \mathrm{CO}_{2}\right)$ y la dureza del agua. El pH del líquido se fija a 6,5 unidades a una temperatura $17^{\circ} \mathrm{C}$. El agua de la red contenía $80 \mathrm{ppm}$ de $\mathrm{Ca}^{++}$. Cuando las barras de error no son visibles, las determinaciones están dentro del intervalo del símbolo

Cuando se añade $\mathrm{CO}_{2}$ no se tienen después de 6-8 horas de tratamiento grandes diferencias por el hecho de aumentar la temperatura de 20 a $50^{\circ} \mathrm{C}$, aunque sí destaca claramente la lentitud con la que se neutraliza la pulpa cuando se trabaja a $10^{\circ} \mathrm{C}$.

Si se emplea $\mathrm{HCl}$ se observa una influencia también beneficiosa al subir la temperatura de $10-20^{\circ} \mathrm{C}$ a $40-50^{\circ} \mathrm{C}$, llegándose a producir en el $\mathrm{pH}$ de la pulpa una diferencia de casi 2 unidades después de 8 horas de tratamiento.

Finalmente, es de resaltar que cuando se emplea $\mathrm{CO}_{2}$, aún trabajando en el caso más desfavorable $\left(10^{\circ} \mathrm{C}\right)$ la neutralización de la pulpa es sensiblemente más rápida que en el mejor de los obtenidos usando $\mathrm{HCl}\left(50^{\circ} \mathrm{C}\right)$, por lo que la ventaja de su empleo resulta evidente.

\section{CONCLUSIONES}

El proceso de neutralización del exceso de álcali, durante la etapa de ennegrecimiento en la elaboración de aceitunas tipo negras, es complejo y en él influyen una serie de variables no estudiadas en detalle hasta ahora. En el presente trabajo se ha demostrado que el tipo de ácido empleado tiene un efecto destacado en la velocidad a que se realiza dicho proceso, siendo mucho más rápido cuando se utiliza anhídrido carbónico que cuando se usa ácido clorhídrico. Asimismo, se ha puesto de manifiesto la
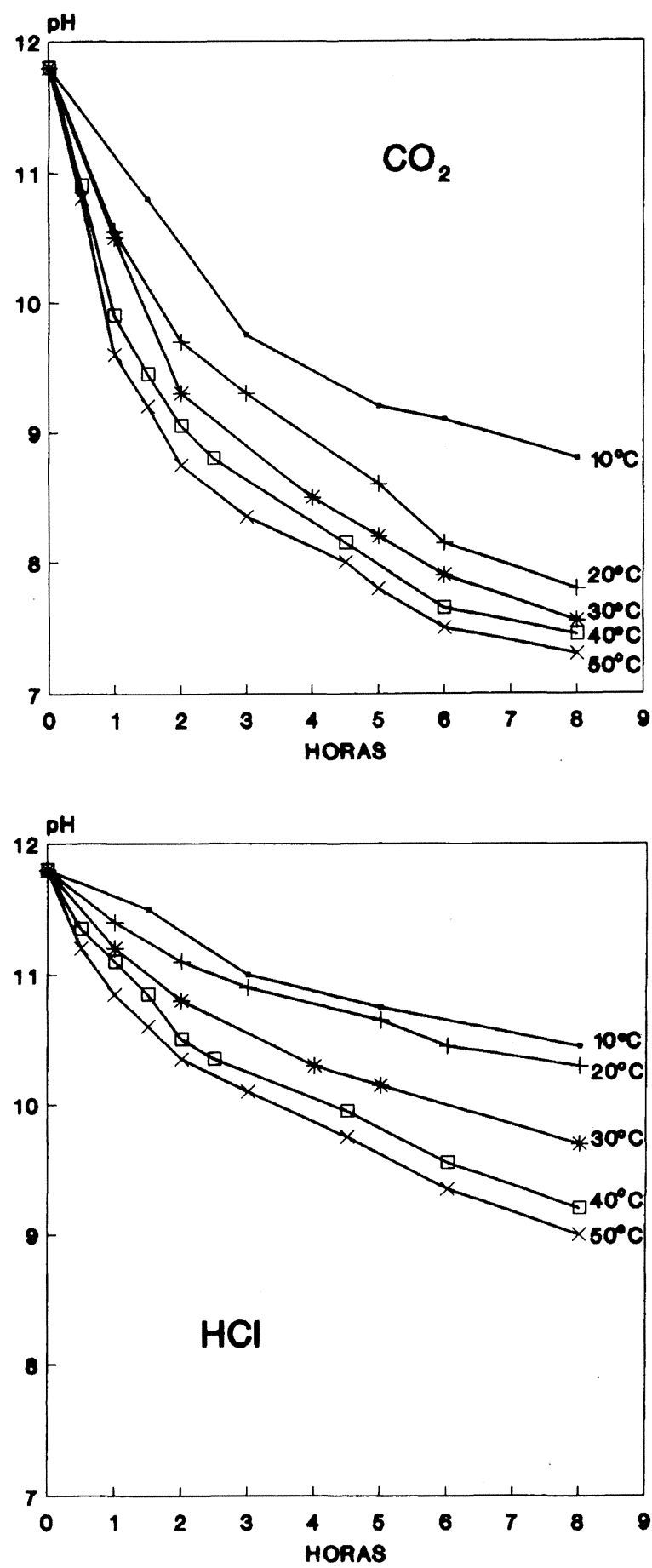

Figura 5.

Evolución del pH de la pulpa de aceitunas a diferentes temperaturas según el agente neutralizante empleado $\left(\mathrm{HCl}\right.$ ó $\left.\mathrm{CO}_{2}\right)$. El lavado se realizó con agua de la red con $35 \mathrm{ppm}$ de $\mathrm{Ca}^{++}$y el pH se fijó a 6,5 unidades.

influencia de la dureza del agua, favoreciéndose la neutralización a medida que se tiene una menor concentración de $\mathrm{Ca}^{++}$, por lo que es aconsejable la utilización de aguas lo menos dura posible.

La velocidad de neutralización aumenta también con la 
temperatura, si bien este efecto no es suficiente para contrarrestar el debido al tipo de ácido, ya que aún a las temperaturas más bajas el anhídrido carbónico siempre ofrece unos resultados más favorables.

\section{AGRADECIMIENTO}

A la CICYT por la financiación del proyecto ALI-911166-CO3-01 y a la firma Carburos Metálicos, S.A. por la colaboración para el desarrollo de este trabajo.

\section{BIBLIOGRAFIA}

Brenes, M.; García, P. y Garrido, A. (1992).- «Phenolic compounds related to the black color formed during the elaboration of ripe olive».-J. Agric. Food Chem. 40, 1192-1196.

Cruess, W.V. (1958).- "Commercial fruit and vegetable products" 4th ed.McGraw-Hill Co, Inc. New York.

Fernández, M.J. y González, F. (1956).-«Cambios en la composición de la aceituna durante su desarrollo II. Acidez y pH del jugo. Determinación de ácidos oxálicos, cítrico y málico" .-Grasas y Aceites 7, 185-189.

Fernández, M.J.; Castro, R.; Garrido, A.; González, F., Nosti, M.; Heredia, A.; Minguez, M.I.; Rejano, L.; Durán, M.C.; Sánchez, F.; García, P. y Castro, A. (1985).- «Biotecnología de la aceituna de mesa».- Instituto de la Grasa y sus Derivados. CSIC. Sevilla.

García, P.; Brenes, M. y Garrido, A. (1991).-«Effect of oxygen and temperature on the oxidation rate during darkening of ripe olives".-J. Food Eng 13, 259-271.

McCorkle, K.C.; Wells, H.M.; Scrimshire H.D. and Beckwith, R.N.-«Method, for processing black ripe olive».- Patent U.S. $N^{\circ} 4,463,023$ (31.7.84).

Miller, M.W. (1979).-«Research on factor affecting the flavor of processed ripe olives".-57th Annual Technical Report of the California Olive Association.-Sacramento, $\mathrm{Ca}$. USA.

Moffat, W.P. (1974).-«Neutralization of caustic efluents».-52 th Annual Technical Report of the California Olive Association".- Sacramento, Ca. USA.

Mondok, F.J. (1979).- "Carbon dioxide gas applications in the olive industry".-57th Annual Technical Report of the California Olive Association. Sacramento, $\mathrm{Ca}$. USA

Rodríguez de la Borbolla, J.M.; Gómez, C.; González, F.; Fernández, M.J.; Gutiérrez, R.; Izquierdo, A.; González, F.; Vázquez, R., y Guzmán, R. (1956).-«El aderezo de aceitunas verdes».-CSIC, Patronato Juan de la Cierva de Investigación Técnica. Madrid.

Scrimshire, H.D. (1987).-«Method for processing black ripe olives».-Patent U.S. $N^{\circ} 4,664,926$ (12.5.87).

(Recibido: Febrero 1993) 